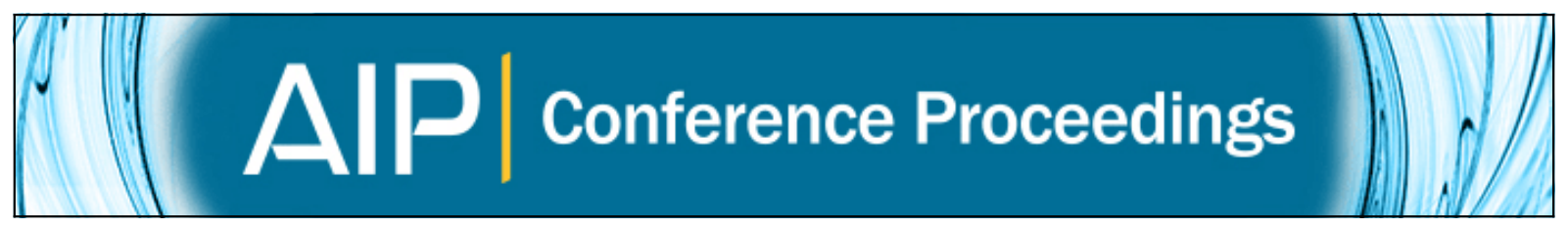

\title{
Screening Surface Contamination with BetaCage
}

R. W. Schnee, Z. Ahmed, S. R. Golwala, D. R. Grant, and K. Poinar

Citation: AIP Conference Proceedings 897, 20 (2007); doi: 10.1063/1.2722063

View online: http://dx.doi.org/10.1063/1.2722063

View Table of Contents: http://scitation.aip.org/content/aip/proceeding/aipcp/897?ver=pdfcov

Published by the AIP Publishing

Articles you may be interested in

Short-lived Rn-222 daughters in cryogenic liquids

AIP Conf. Proc. 1549, 205 (2013); 10.1063/1.4818109

The BetaCage, an ultra-sensitive screener for surface contamination

AIP Conf. Proc. 1549, 132 (2013); 10.1063/1.4818093

Status of BetaCage: an Ultra-sensitive Screener for Surface Contamination

AIP Conf. Proc. 1338, 88 (2011); 10.1063/1.3579564

Beta Cage: A New, Large-Area Multi-Wire Screening Detector For Surface Beta Contamination AIP Conf. Proc. 785, 79 (2005); 10.1063/1.2060456

Radioactive balloon measurements in Utah

Phys. Teach. 35, 478 (1997); 10.1119/1.2344772 


\title{
Screening Surface Contamination with BetaCage
}

\author{
R. W. Schnee*, Z. Ahmed ${ }^{\dagger}$, S. R. Golwala ${ }^{\dagger}$ D. R. Grant* and K. Poinar* \\ ${ }^{*}$ Department of Physics, Case Western Reserve University, Cleveland, OH 44106, USA \\ ${ }^{\dagger}$ Department of Physics, California Institute of Technology, Pasadena, CA 91125, USA
}

\begin{abstract}
Existing screening facilities are insufficiently sensitive to meet the needs of rare-event experiments for lowenergy electron emitters and alpha-decaying isotopes. To provide such screening, the BetaCage will be a low-background, atmospheric-pressure neon drift chamber with unprecedented sensitivity to emitters of low-energy electrons and alpha particles. Minimization of the detector mass and use of radiopure materials reduce background events. The chamber design accepts nearly all alphas and low-energy electrons from the sample surface while allowing excellent rejection of residual backgrounds. A non-radiopure prototype is under construction to test the design. The BetaCage will provide new infrastructure for rare-event science as well as for a wider community that uses radioactive screening for areas including archaeology, biology, climatology, environmental science, geology, planetary science, and integrated-circuit quality control.
\end{abstract}

Keywords: low radioactive background screening, beta decay, alpha decay, dark matter, radioisotope dating, proportional counter PACS: $29.40 . \mathrm{Cs}, 29.40 . \mathrm{Gx}, 91.80 . \mathrm{Hj}, 92.20 . \mathrm{Td} 23.40 .-\mathrm{s}, 23.60 .+\mathrm{e}, 95.35 .+\mathrm{d}$

\section{SCIENTIFIC MOTIVATION AND BASIC DESIGN}

Non-penetrating radioactive contamination on surfaces can provide the dominant background for rare-event searches. The main background for the Cryogenic Dark Matter Search (CDMS [1]) arises from low-energy electrons emitted by radioisotopes on or in the thin films on the detector surfaces. Surface electrons similarly dominate the backgrounds for EDELWEISS [2] and are a significant concern for its proposed successor, EURECA [3]. One leading doublebeta decay experiment, CUORE [4], is currently limited by alpha emission from the radon-daughter ${ }^{210} \mathrm{Po}$. A number of dark matter and neutrino experiments that use liquid targets are similarly sensitive to alpha emission from their containment vessels, due either to occasional misreconstructed events or to resulting loss of livetime. In addition, more sensitive screening is needed to limit alpha emission that produces single-event upsets in integrated circuits, and to allow more sensitive detection of beta-emitting radiotracers used in biology and other fields [5].

Unfortunately, high-purity germanium (HPGe) ionization detectors are insufficient for screening low-energy electrons that do not have associated high-energy gamma-ray emission. Mass spectroscopy is sensitive enough only for some beta-emitting isotopes, is destructive, and usually requires sample processing that may introduce new contaminants or cloud the relation between the intrinsic contamination level and the measured signal. Overall, of the 79 long-lived isotopes that decay by beta emission or electron capture (listed in Table 1 [6]), 27 are inaccessible unless $\alpha$ or $\beta$ screening is employed, or one is able to obtain an extraordinary $1 \mathrm{ppt}$ sensitivity in mass spectroscopy.

Even special-purpose detectors with very thin dead layers (e.g., Si(Li), B-implanted HPGe, or silicon surface-barrier detectors) have deficiencies. There remains a vacuum window that may stop or scatter some particles, backscattering effects will distort the energy spectrum, and it is difficult to obtain the $\sim \mathrm{m}^{2}$ sensitive area desired to obtain high screening throughput and to minimize edge effects. An ideal detector would place the sample directly in a gaseous detection medium to eliminate backscattering and dead-layer effects while providing a large sensitive area. In order to minimize background from ambient penetrating gammas, no more gas would be used than is needed to stop the particles of interest. The detector would have the minimum possible surface area that itself can be a source of background particles. Finally, the detector must provide sufficient spatial information about events to distinguish between those coming from the sample surface and those due to scattering of background particles in the gas, and to measure variations in the contamination level across a sample or across many samples.

Figure 1 shows a sketch of the proposed BetaCage [7], an ultra-low-background drift chamber optimized for detection of $\lesssim 200 \mathrm{keV}$ electrons and alpha particles. Samples are placed in the gas. An open multi-wire proportional counter (MWPC) directly above the sample provides a trigger for particles emanating from the sample. Above this "trigger" MWPC is a large region in which the emitted alphas and betas range out. An electric field in this region drifts the ionization to the top of the chamber, where a second open ("bulk") MWPC collects it. Proportional avalanching in each MWPC provides gain. Crossed grids in both MWPCs provide $x-y$ position determination. The time profile of 
TABLE 1. Detection schemes for all long-lived beta-emitting or electron-capture isotopes. ICP-MS (inductively-coupledplasma mass spectroscopy) is listed both with its commonly achieved sensitivity of $1 \mathrm{ppb}$, and with a better sensitivity of $1 \mathrm{ppt}$, which has been achieved for some isotopes. Of particular note are the isotopes that cannot be screened in any manner except by their emission of beta electrons, and those that can be detected only by their beta emission or by ICP-MS with sensitivity between $1 \mathrm{ppb}$ and $1 \mathrm{ppt}$. A crucial candidate, ${ }^{210} \mathrm{~Pb}$, can be detected only by its emission of alphas or betas.

\begin{tabular}{|c|c|}
\hline Method & Applicable Isotopes \\
\hline ICP-MS (1 ppb) & ${ }^{40} \mathrm{~K}^{48} \mathrm{Ca}^{50} \mathrm{~V}^{87} \mathrm{Rb}^{92} \mathrm{Nb}^{98} \mathrm{Tc}^{113} \mathrm{Cd}^{115} \mathrm{In}^{123} \mathrm{Te}^{138} \mathrm{La}^{176} \mathrm{Lu}^{182} \mathrm{Hf}^{232} \mathrm{Th}^{235} \mathrm{U}^{238} \mathrm{U}^{236} \mathrm{~Np}^{250} \mathrm{Cm}$ \\
\hline $\begin{array}{l}\text { ICP-MS } \\
(1 \mathrm{ppt})\end{array}$ & $\begin{array}{l}{ }^{10} \mathrm{Be}^{36} \mathrm{Cl}^{60} \mathrm{Fe}^{79} \mathrm{Se}^{93} \mathrm{Zr}^{94} \mathrm{Nb}^{97} \mathrm{Tc}^{99} \mathrm{Tc}^{107} \mathrm{Pd}^{126} \mathrm{Sn} \\
{ }^{129} \mathrm{I}^{135} \mathrm{Cs}^{137} \mathrm{La}^{154} \mathrm{Eu}^{158} \mathrm{~Tb}^{166 m} \mathrm{Ho}^{208} \mathrm{Bi}^{208} \mathrm{Po}^{209} \mathrm{Po}^{252} \mathrm{Es}\end{array}$ \\
\hline $\begin{array}{c}\gamma \\
(\mathrm{HPGe})\end{array}$ & 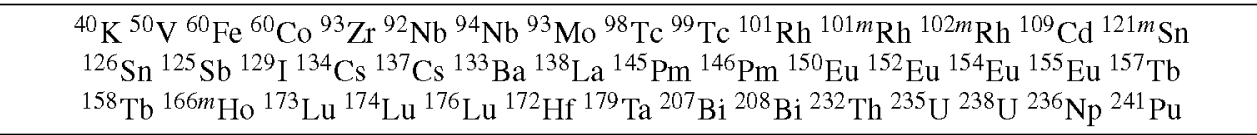 \\
\hline$\alpha$ & ${ }^{210} \mathrm{~Pb}^{208} \mathrm{Po}{ }^{209} \mathrm{Po}{ }^{228} \mathrm{Ra}^{227} \mathrm{Ac}^{232} \mathrm{Th}^{235} \mathrm{U}^{238} \mathrm{U}^{236} \mathrm{~Np}^{241} \mathrm{Pu}{ }^{250} \mathrm{Cm}{ }^{252} \mathrm{Es}$ \\
\hline$\beta /$ ppt MS & ${ }^{10} \mathrm{Be}^{36} \mathrm{Cl}^{79} \mathrm{Se}^{97} \mathrm{Tc}{ }^{107} \mathrm{Pd}^{135} \mathrm{Cs}{ }^{137} \mathrm{La}{ }^{154} \mathrm{Eu}^{209} \mathrm{Po}$ \\
\hline$\beta$ only & ${ }^{3} \mathrm{H}^{14} \mathrm{C}^{32} \mathrm{Si}^{63} \mathrm{Ni}^{90} \mathrm{Sr}^{106} \mathrm{Ru}{ }^{113 m} \mathrm{Cd}^{147} \mathrm{Pm}{ }^{151} \mathrm{Sm}^{171} \mathrm{Tm}{ }^{194} \mathrm{Os}{ }^{204} \mathrm{Tl}$ \\
\hline
\end{tabular}

charge collection in the bulk MWPC determines the spatial profile of the track in the $z$ dimension.

The chamber's design minimizes internal backgrounds and provides excellent rejection of residual and unavoidable backgrounds. By design, the only surface of the detector near the sample is that of the wires, whose area is only a few percent of the sample surface area. Events whose tracks do not originate at the sample or do not terminate inside the drift region will be vetoed using the full 3D information. A third, "veto" MWPC below the sample vetoes throughgoing events. With appropriate construction materials, the limiting background for betas is ejection of electrons from the sample surface by Compton-scattering photons, which can be minimized by external shielding. Alphas should have negligible backgrounds thanks to their uniquely short, dense, straight, high-energy tracks.

The chamber gas and pressure is neon at STP based on desired stopping power, feasibility of purification, drift properties (drift speed, diffusion, electron attachment length), and avalanche gain. Neon's stopping power is large enough that a $30-\mathrm{cm}$-high drift region will contain more than $90 \%$ of $150-\mathrm{keV}$ betas, so the full spectrum of electrons from likely low-energy emitters such as ${ }^{14} \mathrm{C}$ and ${ }^{210} \mathrm{~Pb}$ will be contained in the chamber. Likewise, even a $10-\mathrm{MeV}$ alpha particle will be contained in a 20 -cm-high drift region. Yet the stopping power is low enough that the trigger MWPC can be thick enough $(1 \mathrm{~cm})$ to make assembly straightforward, and the gamma background is sufficiently low in a shield of moderate cost and size. Operation at STP minimizes vacuum system challenges. Neon has no long-lived naturally occurring unstable isotopes, so purification by chemical means is sufficient (in contrast to argon, whose naturally occurring unstable isotope ${ }^{39} \mathrm{Ar}$ would be problematic).

The energy resolution of the BetaCage will be worse than that of $\mathrm{Si}(\mathrm{Li})$ or B-implanted HPGe detectors or the conventional Si barrier detector technology. However, for beta emission spectra, which typically have no sharp features, energy resolution is not critical. The advantage of better sensitivity is more important. Similarly, for alpha screening, the better sensitivity compensates for the loss of diagnostic information.

The BetaCage will also be useful for isotope dating, such as with ${ }^{14} \mathrm{C}$. It is already a common practice to convert organic material for ${ }^{14} \mathrm{C}$ analysis to $\mathrm{CO}_{2}$. Such a gas could be used as the detector medium in the BetaCage if the electron attachment length is long enough; it is expected in the range $30-60 \mathrm{~cm}$. Should electron attachment or the relatively low avalanche gain in $\mathrm{CO}_{2}$ be problematic, converting the carbon source to $\mathrm{CH}_{4}$ used as quench gas in a $90 \%$ neon, $10 \% \mathrm{CH}_{4}$ mixture would work with a $10 \times$ loss in rate. For the background level expected, a sensitivity to $10^{-17}$ is possible if $\mathrm{CO}_{2}$ is used, $10^{-16}$ if $\mathrm{C}$ can be introduced only in the quench gas. With an aggressive effort on the cleanliness of the shield and detector, a sensitivity of 10 times better is possible. Thus, the BetaCage could be more sensitive even than accelerator mass spectrometry, and will be less expensive.

Similarly, to perform dating with tritium, the material may also be converted into the $10 \% \mathrm{CH}_{4}$ quench gas. The BetaCage could reach levels of ${ }^{3} \mathrm{H} / \mathrm{H}=3 \times 10^{-19}$ in a single day's counting, greatly improving the available sensitivity. Such sensitivities are of interest to pollution control agencies: the amount of tritium provides information about general water quality because it measures downward migration of contaminants. Additional studies can be done with the betaemitting isotope ${ }^{210} \mathrm{~Pb}$, used for dating sediment and organisms such as coral, and with very long-lived isotopes ${ }^{10} \mathrm{Be}$ and ${ }^{36} \mathrm{Cl}$, which extend dating to prehistoric times for geology, hydrology, climate, and planetary physics [5]. 


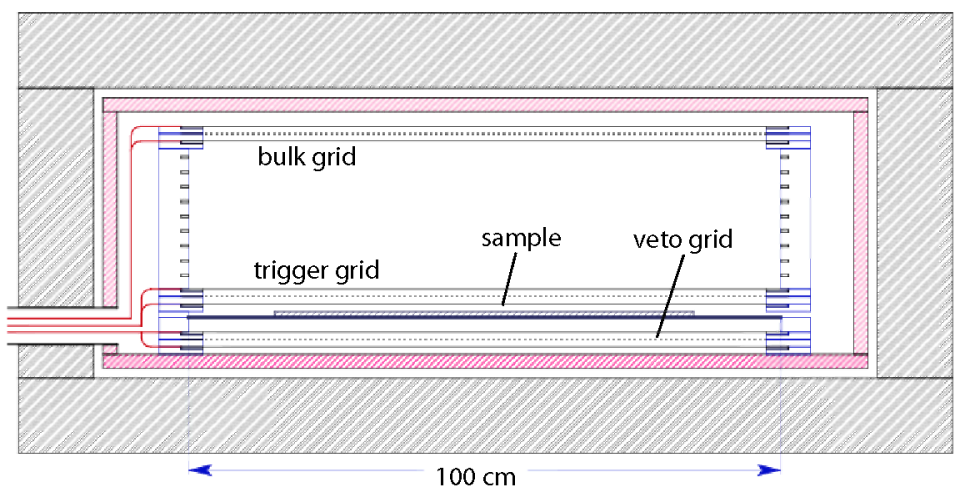

FIGURE 1. Side view of the BetaCage. The trigger, bulk, and veto MWPC grids are indicated, as is the location of the sample. The inner hatched region is the vacuum chamber and the outer hatched region is the shielding.

\section{PROTOTYPE BETA CAGE}

We have designed and begun constructing a non-radiopure version of the BetaCage in order to prototype the design and gain experience with its operation using calibration sources to overcome backgrounds. For the prototype chamber, we will begin with P10 (90\% argon, 10\% methane) because it is inexpensive and well-characterized. Drift and avalanche data for neon is lacking because it is not widely used in drift chambers. After the chamber is commissioned and studied, we will change the gas, first to P5 (95\% argon, 5\% methane) and finally to a 95\% neon, 5\% methane mixture. For initial operation with P10 and P5, we will simply flow through the gas without recovery.

Simulations using MCNP demonstrate that a 40-cm $\times 40-\mathrm{cm} \times 20-\mathrm{cm}$ drift region containing P10 will fully contain $99 \%$ of $156-\mathrm{keV}$ electrons (the endpoint energy of ${ }^{14} \mathrm{C}$, a calibration source). Ranges in neon would be longer, but $100-$ $\mathrm{keV}$ electrons would be fully contained, allowing the use of ${ }^{109} \mathrm{Cd}$ (maximum energy $84 \mathrm{keV}$ ) for calibration. The drift properties of STP P10 are also well-suited to a chamber of this size. A drift velocity of $1-2 \mathrm{~cm} / \mu \mathrm{s}$ is obtained at fields as low at $50 \mathrm{~V} / \mathrm{cm}$ [8]. The diffusion per unit drift length as a function of field for P10 [9] implies negligible rms diffusion, only $\sim 0.25 \mathrm{~cm}$ after a 18 -cm drift length. Finally, electron capture by electronegative impurities is negligible even with 1 part-per-hundred air contamination.

The prototype is a $50-\mathrm{cm} \times 50-\mathrm{cm} \times 20-\mathrm{cm}$ structure that consists of $40-\mathrm{cm} \times 40-\mathrm{cm} \times 1.5-\mathrm{cm}$ trigger and bulk MWPCs separated by a drift field region (see Figure 2). The drift field is set by eighteen 1-mm-thick copper field shapers, separated by 9-mm-thick, ultra-high-molecular-weight polyethylene (UHMWPE) spacers. A 5-mm gap between the top and bottom of the drift field region and the two MWPCs gives an 18-cm-long drift-field region. Individual MWPC grid frames, drift-field shapers, and spacers have the same outer dimensions and are secured together by eight half-inch-diameter Kevlar-coated Nylon rods, with nuts on threaded ends. The assembly's modular design allows individual components to be added and removed with ease. The assembly is housed in an aluminum bell-jar vacuum chamber (shown in Figure 2) to provide control over the detector gas pressure and flow.

Each MWPC has a central anode wire plane and two cathode wire planes with 5-mm spacing between planes, 5-mm wire pitch, and 80 wires in each plane. The wire planes are tensioned and soldered to printed circuit boards mounted to stacked Delrin plastic frames. The anode wires are $25-\mu \mathrm{m}$-diameter, gold-plated tungsten, and the cathode wires are $125-\mu \mathrm{m}$-diameter, gold-plated copper wires. The wires of the cathode plane closer to the drift region run parallel to the anode wires to provide a smooth electric field transition, while the other cathode plane has its wires running crosswise to provide complementary position information. Only the anode planes and the crossed cathode planes will be read out. The wires in each of these planes are grouped into a $35-\mathrm{cm}$-wide central "fiducial" region and an outer "veto" region so events that are not fully contained in the uniform drift field region can be discarded.

The prototype BetaCage will use different high voltages for the drift region, the trigger MWPC anode, the bulk MWPC anode, and the bulk MWPC cathodes to provide full freedom to optimize gains and stability. The high-voltage resistor divider for the drift-field shapers will be soldered directly to the field shapers. A home-built NIM format box contains low-pass filtering for the high voltage and $1 \mathrm{nF}$ HV decoupling capacitors. Roughly 1 meter of HV coaxial cable $(\sim 100 \mathrm{pF})$ will connect the filter/decoupling box to the vacuum chamber HV feedthroughs, carrying the HV into the chamber and the signals out to a NIM-format voltage amplifier. Our calculations yield an anode capacitance of 

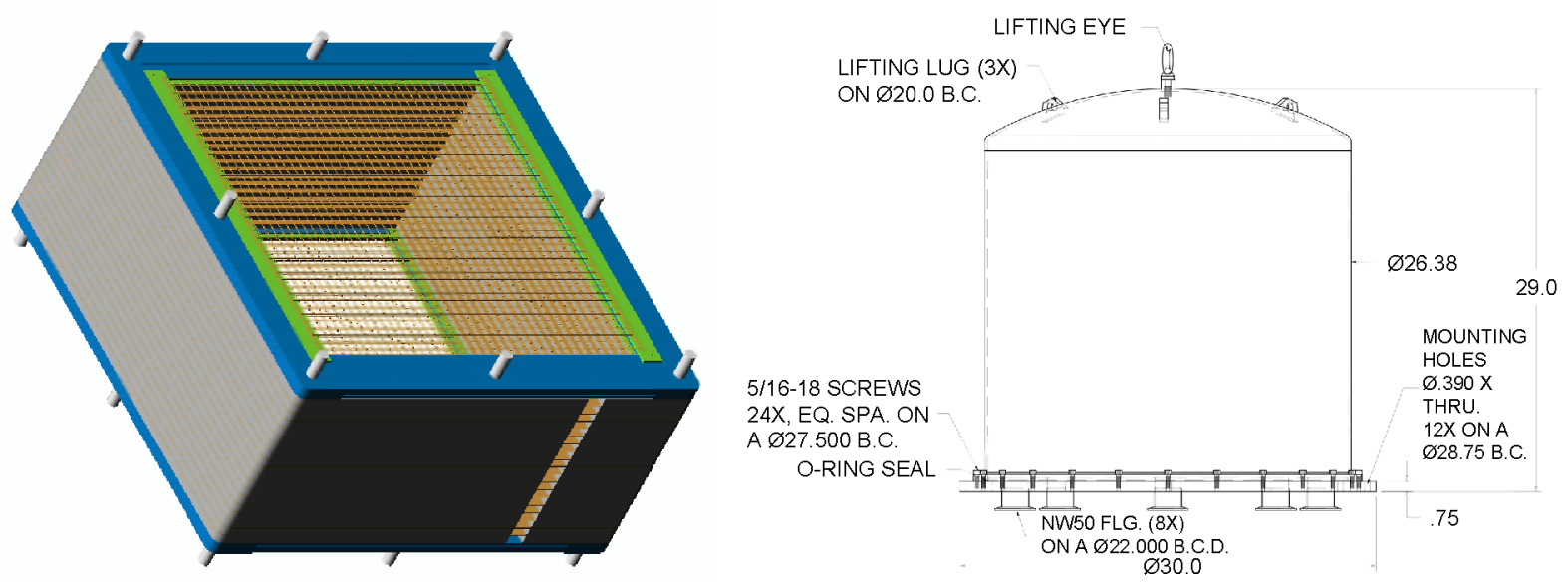

FIGURE 2. Left: CAD drawing of prototype BetaCage. The bulk MWPC is the set of three dark grey frames at the top of the chamber. The trigger MWPC is identical in design and is hidden at the bottom of the drawing. No veto MWPC will be used in the prototype. Right: CAD drawing of bell-jar vacuum chamber. Visible at the bottom are five of the eight NW50 feedthroughs for high-voltage and signal wiring and for gas and vacuum plumbing.

$\sim 2 \mathrm{pF}$ per $40-\mathrm{cm}$-long wire, or about $140 \mathrm{pF}$ total. The wire and cable capacitances initially absorb all the charge that appears on the wire, producing a voltage $Q / C$ with $C=240 \mathrm{pF}$. The charge flows off the capacitances through the decoupling capacitor and across the 50-ohm amplifier input impedance with a decay time $R C=12 \mathrm{~ns}$. The average energy needed to create an ion pair in $\mathrm{Ar}(\mathrm{Ne})$ is $26 \mathrm{eV}(36 \mathrm{eV})$, so we expect about $400(300)$ charges per keV of energy deposited. With a MWPC gain of $10^{4}$, the charge liberated is $65 \mathrm{fC} / \mathrm{keV}$, so the voltage pulse height is $0.3 \mathrm{mV} / \mathrm{keV}$, increasing to $3 \mathrm{mV} / \mathrm{keV}$ after the amplifier. Because the trigger MWPC sees as little as $1-2 \mathrm{keV}$ of the total energy, it will be run at a gain of $10^{5}$, resulting in $30 \mathrm{mV} / \mathrm{keV}$ at the amplifier output.

The amplified bulk MWPC signals will be sent to integrating ADCs. A TDC will determine the time between the trigger and the time of arrival of the first charge at the bulk MWPC so that we can determine whether the track is contained vertically in the chamber. The 50-ohm input impedance of the ADC converts the amplified $3 \mathrm{mV} / \mathrm{keV}$ peak height bulk MWPC signals to a peak current of $60 \mu \mathrm{A} / \mathrm{keV}$. With the $12 \mathrm{~ns}$ pulse decay time constant, the total charge is $0.7 \mathrm{pC} / \mathrm{keV}$. The $\mathrm{ADC}$ calibration is 4 counts $/ \mathrm{pC}$ ( 3 counts $/ \mathrm{keV})$ with $800 \mathrm{pC}$ maximum range $(1.1 \mathrm{MeV})$.

Simple calculations approximating the cathode grids as solid planes indicated the need for $1800 \mathrm{~V}(1500 \mathrm{~V})$ potential difference for the trigger (bulk) MWPC at 5-mm anode-cathode spacing to obtain an avalanche gain of $10^{5}\left(10^{4}\right.$ ). The voltage drop across the drift region was chosen to ensure a smooth transition into the bulk MWPC. We used Ansoft's Maxwell 3D finite-element-modeling software to calculate the electric field structure of the drift region and MWPCs in detail, including the interaction of the grounded vacuum chamber with the fields. The view of the electric potential configuration in a cross-sectional plane (in Figure 3) shows nicely parallel equipotentials inside the drift region, indicating that the proximity of the chamber does not disturb the field configuration and that edge effects are sufficiently small to be isolated using the veto region. Importantly, there are no regions at the corners of the MWPCs with high fields that might cause gas breakdown. The calculated potential in a unit cell of the bulk MWPC, also shown in Figure 3, is sensible: all field lines emerging from the drift region (except on a very thin plane through the center of the cathode and anode wires) terminate on the anode so that essentially all liberated charges will be sensed. Close to the anode wire, the electric field is high enough to ensure the desired proportional gain.

\section{ULTRA-LOW-RADIOACTIVITY BETA CAGE}

The proposed ultra-low-radioactivity BetaCage differs from the prototype version in a number of important ways. First, we will use a neon-methane mixture for the BetaCage instead of a well-characterized argon-methane mixture because argon has a long-lived naturally occurring radioactive isotope, ${ }^{39} \mathrm{Ar}$. Because contamination by ${ }^{14} \mathrm{C}$ in the quench gas is also a consideration, we will reduce the methane fraction to $5 \%$. Since there is little data available on drift and avalanche properties of neon and neon-methane mixtures, we will use the prototype to determine these parameters. 

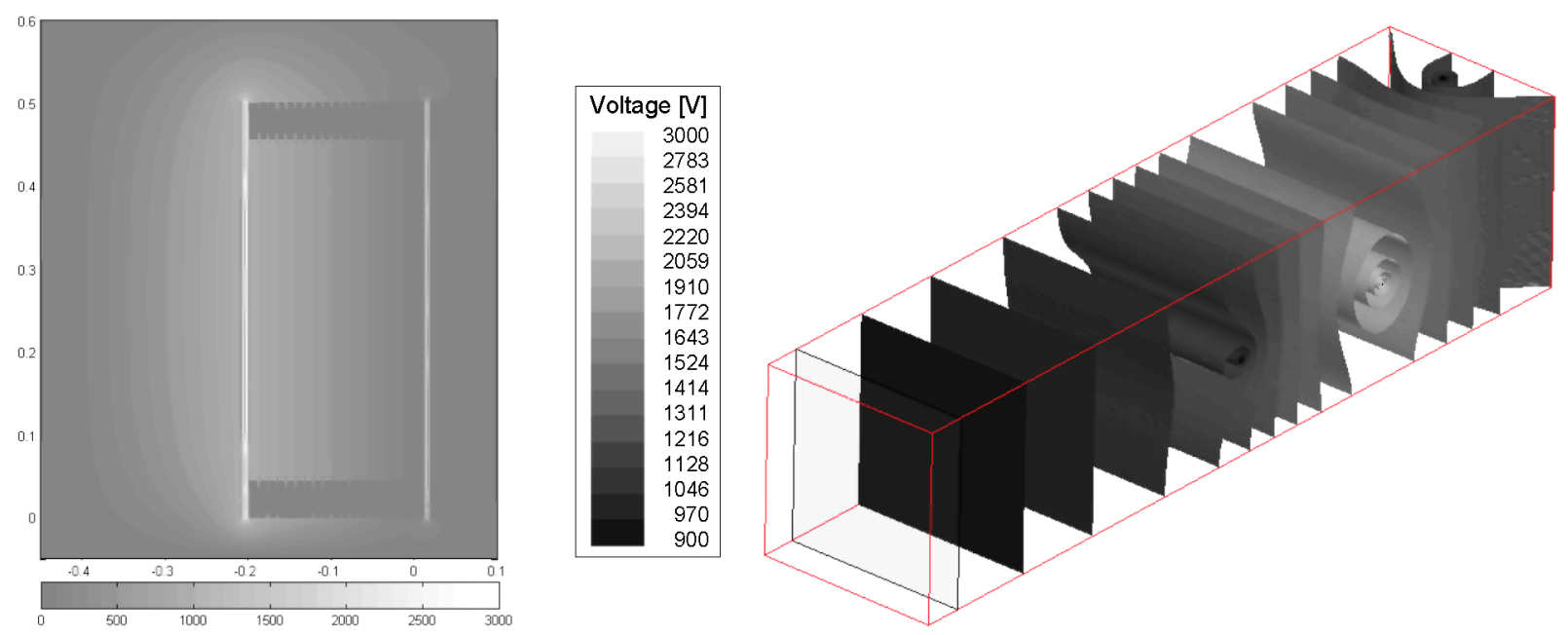

FIGURE 3. Left: Electric potential for a plane bisecting the assembly. The chamber walls are outside the picture, though their influence can be seen in the way the potential tapers to zero in the volume outside the MWPC assembly. The flat bottom of the bell-jar vacuum chamber is at the bottom, causing the compression of the potential in that direction. Right: Electric potential for a unit cell of the MWPCs. The cell is $5 \mathrm{~mm}$ in each transverse dimension and $15 \mathrm{~mm}$ long. From lower left to upper right are the parallel cathode, the anode, and the crossed cathode. The drift region is to the lower left. The surrounding cells meet the four long sides. The upper right extends out of the cage into the empty region of the vacuum chamber.

Neon is not available for purchase pre-mixed with a quench gas, so we will mix the gases from constituent cylinders using one of each gas "in use" and one of each gas held at a lower input pressure to serve as a backup. Gas from each "in use" cylinder will be directed to independent mass-flow-controller transducers. Downstream, a $0.2 \mu \mathrm{m}$ membrane filter followed by a $0.01 \mu \mathrm{m}$ membrane filter will remove particulate contamination. All tubing in the system will be type-316 stainless steel to prevent the ingress of radon.

The cost of neon gas makes it unnecessary to recover the neon from the chamber at the end of each run, but cost-effective to recirculate the neon-methane mixture during running. Since we will not recover the neon, a vacuum chamber (very expensive if radiopure) is not needed. We will design a plastic gas vessel to contain the BetaCage. A getter-stabilized zeolite gas purifier will remove water, $\mathrm{CO}_{2}, \mathrm{O}_{2}$, non-methane hydrocarbons, and particulates greater than $0.003 \mu \mathrm{m}$ in size from the exhausted gas. The neon-methane mixture fraction should remain essentially constant for the days to weeks of counting between gas replacement since the event rate in the chamber will be low enough that dissociation of the methane is negligible.

The drift region of the BetaCage will be $100 \mathrm{~cm} \times 100 \mathrm{~cm} \times 40 \mathrm{~cm}$. It is larger than the prototype to ensure full track containment for electrons as energetic as $156 \mathrm{keV}$, the endpoint of ${ }^{14} \mathrm{C}$. The transverse dimension is a factor of 2.5 larger to increase the available sample area to $\sim 1 \mathrm{~m}^{2}$. The wire spacings of $5 \mathrm{~mm}$ between anode and cathode planes and between individual anode wires or cathode wires will be maintained, so each wire plane will have 200 wires. To minimize alpha backgrounds, we will use stainless steel instead of tungsten wire. Though stainless steel has less tensile strength than tungsten, the critical wire length $(\sim 2.5 \mathrm{~m})$ is still much larger than the chamber.

For the BetaCage, we will seek to discriminate tracks emerging from the sample surface from tracks entering the sample surface based on the full track imaging from digitizing individual wire signals, rather than simply integrating the total charge in the fiducial region. To reduce costs, we will gang the wires together in groups of 10 on the circuit boards to which the wires are attached, reducing the number of amplifiers, digitizers, and high-voltage vacuum feedthrough wires to 20 per wire plane. We can recover the full position resolution of the chamber by creating groups of nonadjacent wires. A randomized ganging pattern will also prevent degeneracies in event position.

Short (1-m) coaxial cables will connect the feedthroughs to a high-voltage filtering/decoupling box similar to that designed for the prototype, though with many more channels. The capacitance per wire will be $2.5 \times$ larger than for the prototype cage, but the number of wires ganged together will be reduced from 70 to 10 , so the capacitance per channel will be lower. The amplified signals will be sent to trigger logic and to waveform digitizers controlled by a PC.

The BetaCage's radiopurity requirements demand changes in some aspects of the construction and materials. The G10 printed circuit boards used in the prototype will be replaced with cirlex (thick kapton). Its radiopurity and its 
mechanical and fabrication properties are understood. We will continue to use plastics such as Delrin and UHWMPE for the frames and for the bolts and dowel pins for aligning and holding the frames. Easily obtained high-radiopurity copper will be used for the field shapers and mechanical support for the frames.

Essential to the ultimate performance of the BetaCage is a low ambient photon background. We will make use of the Soudan Underground Laboratory's Low-Background Counting Facility (LBCF). The LBCF has a 280 square-foot class-10,000 clean room under construction that will provide a $5^{\prime} \times 8^{\prime}$ experimental space at a depth of 2030 meters of water equivalent. The LBCF is surrounded by an active veto shield enclosing the entire $35-\mathrm{m} \times 40-\mathrm{m} \times 100$-m Soudan2 detector hall, with additional veto tubes hung above the clean room itself. The LBCF will provide low-background shielding for the BetaCage that should provide an ambient Ge-equivalent gamma background of $\sim 1 \mathrm{keV}^{-1} \mathrm{~kg}^{-1} \mathrm{day}^{-1}$. The shield will consist of 8 " of lead with a 10-cm thickness of ultra-low-background electroformed copper as the interior lining. To prevent radon exposure, the LBCF will provide purge cabinets for storing samples, a load-lock mechanism for the shielded box for sample changing, and nitrogen gas to keep these spaces Rn-free.

Expected background levels for the BetaCage are discussed in [7]. The expected ambient photon background of $1 \mathrm{keV}^{-1} \mathrm{~kg}^{-1} \mathrm{day}^{-1}$ will yield the dominant background, a rate of $3 \times 10^{-5} \mathrm{keV}^{-1} \mathrm{~cm}^{-2} \mathrm{day}^{-1}$ Compton-scattered electrons ejected from the sample, or $60 \mathrm{~m}^{-2} \mathrm{day}^{-1}$ from 0 to $200 \mathrm{keV}$. The SuperCDMS 1-ton target sensitivity of $10^{-5} \mathrm{keV}^{-1} \mathrm{~cm}^{-2} \mathrm{day}^{-1}$ may thus be easily reached with background subtraction. One day of background measurement would establish the $60 \mathrm{~m}^{-2}$ day $^{-1}$ background rate to a precision of $7.7 \mathrm{~m}^{-2}$ day $^{-1}$, allowing the detection at almost $3 \sigma$ of the rate due to a $10^{-5} \mathrm{keV}^{-1} \mathrm{~cm}^{-2} \mathrm{day}^{-1}$ rate contaminant in a total of 2 days of running (excluding sample installation and purging of the chamber). Fifteen days counting each for sample and background would allow a sensitivity of $4 \times 10^{-6} \mathrm{keV}^{-1} \mathrm{~cm}^{-2}$ day $^{-1}$ rate.

To gain substantially in sensitivity, it will be necessary to reduce the ambient photon background below $1 \mathrm{keV}^{-1} \mathrm{~kg}^{-1} \mathrm{day}^{-1}$, and possibly take additional measures to ensure that internal contaminants remain subdominant. We would need to ensure that the resistors are low-radioactivity or are shielded. Passing the neon through cooled charcoal may be needed to remove radon and krypton (see e.g. [10]). We may need to obtain methane more carefully (the BOREXINO Collaboration analyzed methane with ${ }^{14} \mathrm{C} /{ }^{12} \mathrm{C}<10^{-18}$ [11] but some forms of carbon [12] may have ${ }^{14} \mathrm{C} /{ }^{12} \mathrm{C}$ as high as the $10^{-16}$ value we assume in [7]). We may need to remove radon plating from the wires via etching. These are achievable goals given additional time, funding, and effort.

The prototype chamber should be fully assembled early in 2007 , allowing tests with ${ }^{14} \mathrm{C},{ }^{32} \mathrm{Si}$, and ${ }^{109} \mathrm{Cd}$ sources. We hope to construct the full BetaCage by the end of 2007, with commissioning first on the surface and later at Soudan in 2008-2009. The BetaCage will be immediately useful for screening CDMS detectors. It will have wide applications for screening radioactive surface contaminants to sensitivities better than any existing detector.

RWS and DRG thank the National Science Foundation for financial support under grant no. PHY-0503729. ZA and SRG acknowledge support from the California Institute of Technology.

\section{REFERENCES}

1. D. S. Akerib et al., Phys. Rev. D 72, 052009/1-38 (2005).

2. P. C. F. Di Stefano et al., J. Phys. Conf. Ser. 39, 70-74 (2006).

3. H. Kraus et al., J. Phys. Conf. Ser. 39, 139-141 (2006).

4. R. Ardito et al., Prog. Part. Nucl. Phys. 57, 203-216 (2006).

5. P. Cushman, Report of the DUSEL Working Group on Low Background Counting Facilities and Prototyping for the NSF S1 Solicitation White Paper, to be posted on dusel.org.

6. R. W. Schnee et al., "The SuperCDMS experiment," in Dark Matter in Astro- and Particle Physics: Proceedings of the International Conference Dark 2004, College Station, edited by H. V. Klapdor-Kleingrothaus, and R. Arnowitt, Springer-Verlag, Heidelberg, 2005, pp. 259-268, astro-ph/0502435; H. Nelson, private communication.

7. T. Shutt, C. E. Dahl, L. de Viveiros, R. J. Gaitskell, and R. W. Schnee, "Beta Cage: A New, Large-Area Multi-Wire Screening Detector For Surface Beta Contamination," in AIP Conf. Proc. 785: Topical Workshop on Low Radioactivity Techniques: LRT 2004, edited by B. Cleveland, R. Ford, and M. Chen, American Institute of Physics, Melville, NY, 2005, pp. 79-83.

8. B. Jean-Marie, V. Lepeltier, and D. L'Hote, Nucl. Instrum. Meth. 159, 213-219 (1979); W. Blum, and L. Rolandi, Particle Detection with Drift Chambers, Springer-Verlag, Berlin, 1993.

9. Johns Hopkins University, Lawrence Berkeley Laboratory, U. C. Riverside, and Yale University, "Proposal for a PEP facility based on the time projection chamber," SLAC-Pub-5012 (1976).

10. D. N. McKinsey, and K. J. Coakley, Astropart. Phys 22, 355-368 (2005); C. Arpesella et al., Astropart. Phys 18, 1-25 (2002).

11. R. P. Beukens, Annual Report, IsoTrace Laboratory, Canadian Centre for Accelerator Mass Spectroscopy at the University of Toronto, University of Toronto, Toronto, 1992.

12. G. Bonvicini, N. Harris, and V. Paolone, "The chemical history of ${ }^{14} \mathrm{C}$ in deep oilfields," hep-ex/0308025 (2003). 